\title{
Potenciales aplicaciones de películas de quitosano en alimentos de origen animal: una revisión.
}

\author{
Carolina Valenzuela V. ${ }^{1}$ José Ignacio Arias. $^{2}$ \\ ${ }^{1}$ Departamento de Fomento de la Producción Animal. Facultad de Ciencias Veterinarias y Pecuarias. \\ Universidad de Chile. \\ ${ }^{2}$ Departamento de Ciencias Clínicas. Facultad de Ciencias Veterinarias y Pecuarias. Universidad de Chile. \\ Dirección de Investigación Facultad de Ciencias Veterinarias y Pecuarias. Santa Rosa 11.735, La Pintana. \\ Casilla 2, correo 15, La Granja. Email: cvalenzuelav@u.uchile.cl
}

\begin{abstract}
Resumen
En la actualidad se ha observado un incremento constante del número de intoxicaciones y muertes humanas alrededor del mundo debido a la contaminación de los alimentos de origen animal con patógenos perjudiciales para la salud. Es por esto que varias tecnologías se han investigado, desarrollado y aplicado para mejorar la inocuidad alimentaria. Dentro de estos métodos cabe mencionar la utilización de bioembalajes con polímeros naturales con una alta actividad antimicrobiana, como el quitosano, obtenido a partir de la desacetilación alcalina de la quitina. El quitosano presenta una gran variedad de propiedades biológicas como biodegradabilidad, biocompatibilidad, baja toxicidad, actividad antimicrobiana de amplio espectro, y capacidad de formación de películas comestibles. Esto lo convierten en un biomaterialatractivo para aplicaciones biotecnológicas e industriales. Un ejemplo de esto son las películas de quitosano,que hansido probadas en conservación de alimentos y la tecnología de envasado, ya que exhiben una alta actividad contra patógenos, como hongos, levaduras,bacterias Gram-positivas y negativas, disminuyendo el deterioro de los alimentos de origen animal y vegetal. Este artículo revisalas potenciales aplicaciones del quitosano debido a su acción antimicrobiana sobre los principales patógenos que afectan la inocuidad de los productos de origen animal como carne, cecinas, embutidos, leche, huevos, queso, pescado y mariscos, que repercuten en la mejora de la calidad y el aumento de la vida útil de éstos productos.
\end{abstract}

Palabras clave: quitosano, alimentos, origen animal, antimicrobiano, inocuidad.

\section{Introducción}

La seguridad alimentaria sigue siendo un problema crítico de salud pública debido a los constantes brotes de enfermedades transmitidas por los alimentos en el mundo (Käfersteinet al., 1997).En los últimos años se ha descrito un aumento de las intoxicaciones alimentarias, teniendo una destacada participación las producidas por alimentos de origen animal. Esto puede atribuirse en cierta parte por el aumento del comercio mundial y exportación de productos alimenticios, los cambios en la producción actual de alimentos, el impacto de estilos de vida modernos, los cambios en el consumo de alimentos y la aparición de nuevos patógenos (Collins, 1997; Tauxe, 1997). 
Según la Organización de la Naciones Unidas para la Alimentación y la Agricultura y la Organización Mundial de la Salud, la contaminación de los alimentos con microorganismos patógenos provoca enormes pérdidas en la industria alimentaria y la economía (FAO/WHO, 2003), por tanto, diversos procesos químicos, físicos y nuevas tecnologías han sido desarrollados para extender la vida útil de los alimentos. Entre tales procesos, el envasado de los productos alimenticios es un factor fundamental en su conservación y comercialización. Además como el crecimiento microbiano en la superficie de los alimentos es la principal causa de deterioro de éstos y de transmisión de enfermedades, el concepto de usar un recubrimiento activo comestible y biodegradable para inhibir el deterioro y la patogenicidad de los microorganismos ha recibido un interés considerable en el último tiempo (Ouattara et al., 2000; Coma et al., 2001; Jeon et al., 2002). En este contexto, las películas y soluciones de quitosano han demostrado ser una gran promesa para su aplicación como conservantes de alimentos de origen animal (No et al., 2007; Dutta et al., 2009).

El quitosano es un hetero polisacárido que se encuentra en casi un tercio del total de la pared celular de algunos hongos (Agullóet al., 2003), sin embargo, su principal fuente de obtención es mediante la desacetilación alcalina de la quitina (Majeti y Kumar, 2000). La quitina es el polímero natural más abundante luego de la celulosa, con la que guarda similitudes estructurales y como ella, funciona como un polisacárido estructural (Tolaimateet al., 2000(Arias et al., 2008)). La quitina es un homo polímero formado por unidades 2acetamido-2-desoxi-D-glucopiranosa (GlcNAc, Nacetil glucosamina) unidas por enlaces glicosídicos $\beta$ $(1 \rightarrow 4)$, y constituye la mayor fuente de contaminación en las zonas costeras. El quitosano es el derivado Ndesacetilado de la quitina. La reacción de desacetilación, es decir, la pérdida del grupo acetilo del grupo amida del carbono 2, da lugar a un grupo amino en esa posición, y cuando la desacetilación del material de partida es incompleta se obtiene una mezcla de cadenas que contienen residuos de unidades GlcNAc y 2-amino-2-desoxi- $\beta$-Dglucopiranosa (GlcN) unidos por enlaces glucosidicos $\beta(1 \rightarrow 4)$, cuya relación depende de las condiciones de reacción y que genera quitosanoscon distintas propiedades estructurales, físicas, químicas, y funcionales (Majeti y Kumar, 2000).

Estructuralmente se pueden distinguir dos tipos de quitosano (Q) principalmente, $\alpha-\mathrm{Q}$ y $\beta-\mathrm{Q}$, derivados desde $\alpha$ y $\beta$ quitinas, respectivamente, las cuales tienen una estructura y origen diferentes. La $\alpha$-quitina presenta cadenas paralelas y la $\beta$-quitina, antiparalelas, a lo largo de su estructura lineal. La primera se encuentra presente en la cutícula de crustáceos (cangrejos, camarones, langostinos, langostas, jaibas, etc.), y en menor proporción en la cutícula de hongos, levaduras e insectos (Tan et al., 1996; Rabea et al., 2003; Tharanathan y Kittur, 2003). La segunda ( $\beta$ quitina) es obtenida desde la pluma cartilaginosa interna del endoesqueleto de calamares, como el calamar gigante (Dosidicus gigas), más conocido como jibia en Chile o pota en Perú (Subasingle, 1995). La fuente y el método de obtención determinan la composición de las cadenas de quitosano, su grado de desacetilación y el peso molecular promedio, que son parámetros de obligado conocimiento para la caracterización de este polímero, ya que influyen sobre sus propiedades fisicoquímicas, biológicas $\mathrm{y}$ funcionalidad (Rhaziet al., 2004).

El quitosano es insoluble en agua y tiene la propiedad de formar películas por sí solo (Jeonet al., 2002), a partir de soluciones diluidas en ácidos orgánicos (Begin y Van Calsteren, 1999), mediante variadas metodologías siendo la más utilizada evaporación del solvente y moldeado (Butler et al., 1996; Caneret al., 1998). En este contexto el quitosano posee propiedades únicas que lo hacen un ingrediente ideal para el desarrollo de películas comestible y su aplicación en sistemas alimentarios como agente antimicrobiano, ya que presenta una baja toxicidad, con un $\mathrm{DL}_{50}=16$ $\mathrm{ml} / \mathrm{kg}$, valor que sitúa al quitosano en un nivel similar a la azúcar y lo hace menos tóxico que la sal (Romanazziet al., 2002). Por otra parte, es deseable que los polímeros formadores de películas sean biodegradables y que no generen productos dañinos como resultado de su degradación. Algunos estudios han demostrado que la quitina y el quitosano se biodegradan in vivo debido a su susceptibilidad a la hidrólisis enzimática de los enlaces $\beta(1 \rightarrow 4)$ mediada 
por la enzima lisozima, presente en el organismo humano (Sashiwaet al., 1990; Shigemasaet al., 1994). Sus productos de degradación son oligosacáridos o monosacáridos, metabolitos naturales que una vez absorbidos pueden ser incorporados a las rutas metabólicas de glicoaminoglicanos o glicoaminoproteínas, o bien, excretados (Dinesh y Alok, 2000). Las películas de quitosano son bioadherentes (Henriksenet al., 1996), altamente biocompatibles (Raafat y Sahl, 2009), transparentes e incoloras (Wileset al., 2000; Park et al., 2002), lo que facilita su aplicación en la superficie de los alimentos.

Las películas de quitosano han demostrado tener un efecto antimicrobiano de amplio espectro, que ha sido explicado a través de varios mecanismos de acción, contra microorganismos que comúnmente contaminan alimentos de origen animal como bacterias del tipo Escherichiacoli, Listeria monocytogenes, Pseudomonasspp, Salmonelosis, Staphylococcusaureus, entre la principales,identificadas como los más potentes patógenos asociados con enfermedades alimentarias (Mead, 1999; Huang et al., 2012). Por tanto, el quitosano se ha convertido en un envase prometedor para evitar tanto la contaminación de patógenos como el crecimiento demicroorganismos causantes de deterioro en la superficie de los alimentos de origen animal, materia que ha sido sujeta de variadas investigaciones. Además en el presente artículo se revisarán los mecanismos de acción antimicrobianos y sus alcances aplicativos a los diferentes alimentos de origen animal.

\section{Desarrollo:}

\subsection{Aplicaciones del quitosano como antimicrobiano en productos de origen animal}

El quitosano como agente antimicrobiano presenta varias ventajas sobre otros tipos de envases activos, ya que posee una mayor actividad antibacteriana, un espectro de acción más amplio, una tasa de inhibición mayor, y menor toxicidad para las células de mamíferos (Franklin y Snow, 1981).En general se reconoce que levaduras y mohos son los microorganismos más sensible al quitosano, seguido por bacterias Gram-positivas y Gram-negativas (Aideret al., 2010). Las propiedades antimicrobianas de soluciones y películas de quitosano han sido reportadas en varios estudios, en donde este biopolímeroha mostrado una gran capacidad de disminuir la multiplicación de una amplia variedad de bacterias principalmente, presentes en alimentos de origen animal (Tabla 1).

Tabla 1.Actividad antimicrobiana del quitosano sobre microorganismos contaminantes de alimentos de origen animal.

\begin{tabular}{lll}
\hline Microrganismo & Alimento & Referencia \\
\hline Bacterias & & \\
Acinetobacterbaumannii & Huevos & Leleuet al. (2011) \\
\hline Aeromonashydrophila & Tocino y kababs de cordero & Raoet al.(2005) \\
Alcaligenessp & Huevos & Leleuet al. (2011) \\
Bacillus cereus & Carne de vacuno & Darmadji y Izumimoto (1994) \\
Bacillus subtilis & Carne de vacuno & Darmadji y Izumimoto (1994) \\
& Carne de cerdo & Park et al. (1999) \\
Bacteriasácidolácticas & Salchicha & Raoet al. (2005), Younet al. (2000) \\
& Hamburguesa de bacalao & López-Caballero et al. (2005) \\
Brochothrixthermosphacta & Salchicha & Georganteliset al. (2007) \\
& Carne de cerdo & Lee et al. (2003) \\
Carnobacteriumsp & Carne de pollo & Petrouet al. (2012) \\
Clostridiumhistoryticum & Salchicha & Leleuet al. (2011) \\
Clostridiumperfringens & Salchicha & Younet al. (2001) \\
\hline
\end{tabular}




\begin{tabular}{lll}
\hline & Carne molida (vacuno o & Junejaet al. (2006) \\
pavo) & Carne de vacuno & Darmadji y Izumimoto (1994) \\
Entiformes & Queso mozzarella & Altieriet al. (2005) \\
& Pastrami & Ouattaraet al. (2000) \\
& Hamburguesa de bacalao & López-Caballero et al. (2005) \\
& Salchichas & Georganteliset al. (2007) \\
& Carne de pollo & Petrouet al. (2012) \\
Escherichiacoli & Carne de vacuno & Darmadji y Izumimoto (1994) \\
& Salchichas & Park et al. (1999), Younet al. (2000, \\
& Carne de cerdo & 2001) \\
& Tocino y kababs de cordero & Lee et al. (2003) \\
& Filete de bacalao & Raoet al. (2005) \\
& Huevos & Gómez-Estaca et al. (2010) \\
& & Leleuet al. (2011) \\
EscherichiacoliO157 & Jugo de pollo & Lahmeret al. (2012) \\
Gram-positivas y negativas & Carne de cordero y salame & Kanattet al. (2008) \\
Lactobacillusacidophilus & Filete de bacalao & Gómez-Estaca et al. (2010) \\
Lactobacillus curvatus & Carne de cerdo & Lee et al. (2003) \\
Lactobacillus plantarum & Carne de vacuno & Darmadji y Izumimoto (1994) \\
& Carne de cerdo & Lee et al. (2003) \\
Lactobacillus viridescens & Carne de cerdo & Sagooet al. (2002) \\
Listeria innocua & Salchichas & Younet al. (2001) \\
& Carne de cerdo & Lee et al. (2003), Sagooet al. (2002) \\
& Filete de bacalao & Gómez-Estacaet al. (2010)
\end{tabular}

\begin{tabular}{lll} 
Listeria monocytogenes & Salchichas & Park et al. (1999), Younet al. (2000) \\
& Carne asada & Beverlyaet al. (2008) \\
& Jamón & Ye et al. (2008a) \\
& Salmón ahumado & Ye et al. (2008b) \\
& Sopa de pescado & Fernandez-Saiz et al. (2010) \\
& Paté de vacuno & Albuquerque et al. (2011) \\
& Huevos & Leleuet al. (2011) \\
Micrococci & Carne de vacuno & Darmadji y Izumimoto (1994) \\
Micrococcusvarians & & Gómez-Estacaet al. (2010) \\
Photobacteriumphosphoreum & Filete de bacalao & Lee et al. (2003) \\
Pseudomonas aeruginosa & Carne de cerdo & Park et al. (1999), Younet al. (2000) \\
& Salchichas & Ha y Lee (2001) \\
Pseudomonas fluorescens & Leche & Gómez-Estaca et al. (2010) \\
& Filete de bacalao & Darmadji and Izumimoto (1994), \\
Pseudomonasspp. & Carne de vacuno & Lee et al. (2003) \\
& Carne de cerdo & López-Caballero et al. (2005) \\
& Hamburguesa de bacalao & Raoet al. (2005) \\
& Tocino y kababs de cordero & Georganteliset al. (2007) \\
\hline
\end{tabular}




\begin{tabular}{|c|c|c|}
\hline & $\begin{array}{l}\text { Huevos } \\
\text { Carne de pollo }\end{array}$ & $\begin{array}{l}\text { Leleuet al. (2011) } \\
\text { Petrouet al. (2012) }\end{array}$ \\
\hline Salmonella Enteritidis & $\begin{array}{l}\text { Carne de cerdo } \\
\text { Salchicha }\end{array}$ & $\begin{array}{l}\text { Lee et al. (2003) } \\
\text { Park et al. (1999), Younet al. (2000, } \\
\text { 2001) }\end{array}$ \\
\hline Salmonella spp. & Sopa de pescado & Fernandez-Saiz et al. (2010) \\
\hline Salmonella Typhimurium & $\begin{array}{l}\text { Carne de cerdo } \\
\text { Salchichas } \\
\text { Huevos }\end{array}$ & $\begin{array}{l}\text { Lee et al. (2003) } \\
\text { Park et al. (1999), Younet al. (2000, } \\
\text { 2001) } \\
\text { Leleuet al. (2011) }\end{array}$ \\
\hline Serratialiquefaciens & Jamón & Park et al. (1999), Younet al. (2000) \\
\hline Serratiamarcescens & Huevos & Leleuet al. (2011) \\
\hline Shewanellaputrefaciens & Filete de bacalao & Gómez-Estacaet al. (2010) \\
\hline Staphylococcus aureus & $\begin{array}{l}\text { Carne de vacuno } \\
\text { Salchichas } \\
\text { Tocino y kababs de cordero } \\
\text { Hamburguesa de bacalao } \\
\text { Ostras crudas } \\
\text { Sopa de pescado }\end{array}$ & $\begin{array}{l}\text { Darmadji y Izumimoto (1994) } \\
\text { Park et al. (1999), Younet al. (2000) } \\
\text { Raoet al. (2005) } \\
\text { López-Caballero et al. (2005) } \\
\text { Chhabraet al. (2006) } \\
\text { Fernandez-Saiz et al. (2010) }\end{array}$ \\
\hline Staphylococcus warneri & Huevos & Leleuet al. (2011) \\
\hline Levaduras & & \\
\hline Saccharomycodesludwigii & Carne de cerdo & Sagooet al.(2002) \\
\hline
\end{tabular}

Debido a su amplio espectro de acción,ya sea en forma de solución, película comestible, polvo agregado directamente en la preparación de alimentos procesados u otras formulaciones,se ha utilizado como preservante en la industria de alimentos de origen animal, en donde se aprecia que el quitosano es capaz de inhibir y/o disminuir el crecimiento de los principales microorganismos patógenos de alimentos de origen animal. Sin embargo, además de su acción antimicrobiana en alimentos, se ha descrito su acción in vitro sobre patógenos contaminantes de productos cárnicos y cecinas mencionando entre los principales: Escherichiacoli O157:H7 (Yang et al., 2007), causante de graves complicaciones como la colitis hemorrágica y el síndrome urémico hemorrágico, y que su mayor fuente de infección son los alimentos de origen animal, principalmente carne de vacuno molida y leche cruda (Doyle, 1991; Smith, 1997); Escherichiacoli (Li et al., 2006; Xinget al., 2009); Staphylococcusaureus (Li et al., 2006; Liuet al., 2006; Xinget al., 2009); Listeria monocytogenes (Zivanovicet al., 2004; Li et al., 2006); Bacilluscereus(Mellegårdet al., 2011).Otro factor crucial a considerar en el efecto del quitosano para generar una inhibición o disminución de la carga microbiana es la concentración usada, esta depende de varios factores inherentes al quitosano y su preparación, y del microrganismo. Wang (1992) observó que una concentración alta de quitosano (1$1.5 \% \mathrm{p} / \mathrm{v}$ ) se requiere para la inactivación completa de Staphylococcusaureus después de dos días de incubación a $\mathrm{pH}$ 5,5. Bacilluscereus requiere una concentración de quitosano del orden de $0,02 \% \mathrm{p} / \mathrm{v}$ para un efecto bactericida.Escherichiacoli y Proteusvulgaris mostraron crecimiento mínimo en soluciones de quitosano al $0,005 \%$, y una inhibición completa a $>0,0075 \%$ de quitosano (Simpson et al., 1997).

Sin embargo, hay otros autores que describen resultados diferentes, debido a que la actividad antimicrobiana del quitosano y su concentración mínima inhibitoria varía considerablemente con el tipo de quitosano ( $\alpha, \beta$-quitosano), grado de desacetilación (Tsaiet al., 2002), peso molecular (Jeonet al., 2001; No et al., 2002), organismo blanco y las condiciones del medio en el que se aplica, particularmente $\mathrm{pH}$, fuerza iónica y presencia de solutos susceptibles de reaccionar con quitosano a través de interacciones 
intermoleculares, que puedan bloquear parcial o completamente la reactividad del grupo amino activo (No et al., 2002).

Por otra parte,soluciones de quitosano con diferentes pesos moleculares, y oligosacáridos obtenidos a partir de la hidrólisis enzimática han demostrado tener un efecto inhibitorio contra Campylobacter (Ganan et al., 2009; Mengíbaret al., 2011), agente que se considera como el más importante patógeno en alimentos de origen animal e involucrado en la transmisión de enfermedades gastrointestinales en todo el mundo, transmitido principalmente por las aves de corral, y en especial el pollo, siendo ésta la principal fuente de infección en los seres humanos (Lee y Newell, 2006). Dado que una gran proporción de la producción de pollo de la Unión Europea está contaminado con Campylobacter (EFSA, 2010), y también de acuerdo a la reciente prohibición por la Unión Europea del uso de antibióticos en la alimentación animal para promover el crecimiento (European Commission, 2003), es esencial la búsqueda de nuevos productos y estrategias naturales sostenibles para reducir la incidencia de contaminación microbiana en la cadena alimentaria. Por tanto, debido a la abundancia, versatilidad y facilidad de aplicación del quitosano, este biopolímero se convierte en un compuesto prometedor para su aplicación como agente antimicrobiano en alimentos de origen animal.

En relación a estudios realizados en leche y productos lácteos existen pocos reportes en la literatura, posiblemente por el hecho que el quitosano podría inhibir microorganismos necesarios para la elaboración de productos lácteos fermentados como Streptococcusthermophilus $\quad y$ Lactobacillusdelbrueckiissp. ,Bulgaricus, otras bacterias ácido lácticas, propinibacterias etc. Sin embargo, hay literatura del efecto del quitosano sobre la contaminación con microorganismos en el proceso de post-producción de la leche. Ha y Lee (2001) investigaron la efectividad de soluciones de quitosano $(0,03 \% \quad \mathrm{p} / \mathrm{v})$ para minimizar la contaminación microbiana (bacterias y levaduras) en leche procesada. Observando una completa inhibición del crecimiento microbiano en leche con sabor a plátano durante 15 días de almacenamiento a 4 y $10^{\circ} \mathrm{C}$.

\subsection{Mecanismos de acción antimicrobiana del quitosano}

La actividad antimicrobiana del quitosano difiere del microorganismo, en el caso de los hongos, este polímero ejerce una efecto antifúngico inhibiendo la formación de esporas e hifas (Hernández-Lauzardoet al., 2008). En contraste, la actividad antibacteriana del quitosano es más compleja y difiere entre bacterias Gram-positivas y Gram-negativas debido a la diferente composición de la superficie celular entre ambos tipos de bacterias. Respecto al mecanismo de acción antimicrobiana del quitosano no está totalmente dilucidado, y dentro de los mecanismos propuestos, los investigadores coinciden que posee tres posibles acciones.

\section{Carácter catiónico}

El primer mecanismo se basa en las cargas positivas que posee el quitosano $\left(\mathrm{NH}_{3}{ }^{+}\right)$, debido a la presencia de un cambio sobre el C-2 del monómero de la glucosamina, cuando este polímero se solubiliza en soluciones ácidas con $\mathrm{pH}$ menor a su $\mathrm{pKa}(\mathrm{pH} \approx 6-7)$ (Figura 1).

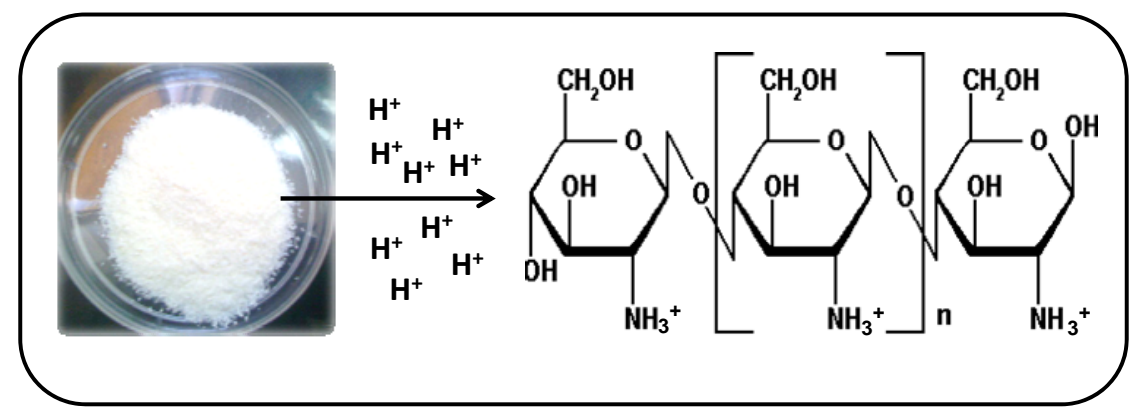

Figura 1.Estructura y carácter catiónico del quitosano en disolución ácida. 
Para el caso de soluciones de quitosano llevadas a $\mathrm{pH}$ 5,5 se ha estimado que los grupos aminos se encuentran parcialmente protonados. En cambio a pHs menores éstos grupos se encuentran protonados (Helanderet al., 2001; No et al., 2007), reaccionando potentemente con los grupos hidrofílicos aniónicos (tales como lipopolisacárido, ácido teicoico de las bacterias Gram-negativas y proteínas celulares específicas),que juegan un papel primordial en la actividad antibacteriana de las membranas celulares de los microorganismos (Rabea et al., 2003). Por ejemplo, en estudios realizados en algunas levaduras se reportó que la parte externa de la membrana plasmática esta enriquecida por esfingolípidos, que están cargados negativamente, los cuales interactuaban con los grupos amino del quitosano, generando una desestabilización de la membrana y lisis celular (Zakrzewskaet al., 2005). El quitosano forma canales de transporte de moléculas en bicapas lipídicas artificiales, lo que provee evidencia que el quitosano puede desorganizar la membrana celular (Zakrzewskaet al., 2007).Liuet al. (2004) determinaron que la acción antimicrobiana del quitosano consistía en la destrucción de las membranas internas y externas de las bacterias, liberando los componentes intracelulares. Helanderet al. (2001) reportaron que el quitosano cambia el estado de la membrana externa y modifica la superficie celular, generando el debilitamiento de la función celular de bacterias Gram-negativas. Otro factor que incide en la actividad microbiana del quitosano es la fase de crecimiento en la que se encuentra el microorganismo, ya que la electronegatividad de la membrana cambia en las diferentes etapas del crecimiento bacteriano, lo que puede generar menor o mayor susceptibilidad (Tsai y $\mathrm{Su}, 1999$; Yang et al., 2007).

\section{Agente quelante}

Un segundo mecanismo propuesto para el quitosano, es que puede actuar como agente quelante, formando complejos con metales trazas (incluyendo $\mathrm{Ni}^{2+}, \mathrm{Zn}^{2+}$, $\mathrm{Co}^{2+}, \mathrm{Fe}^{2+}, \mathrm{Mg}^{2+} \mathrm{y} \mathrm{Cu}^{2+}$ ) en condiciones ácidas, inhibiendo el desarrollo microbiano y producción de toxinas, y la disponibilidad de micronutrientes esenciales para las actividades celulares vitales de los microorganismos (Rabea et al., 2003). También se ha observado que el quitosano es capaz de quelar cationes divalentes estableciendo interacciones electroestáticas con moléculas aniónicas (fosfato, carboxilato), que componen los lipopolisacáridos, desestabilizando la membrana celular de las bacterias (Helanderet al., 1997).

\section{Penetración al interior de la célula}

Como tercer mecanismo se ha postulado que oligómeros de quitosano de bajo peso molecular penetran en las células de los microrganismos e impiden el crecimiento de éstas, ya que inhibe la acción de varias enzimas e interfiere en la síntesis de proteínas por inhibición de la transformación de ADN en ARNm(Tharanathan y Kittur, 2003).

En resumen la actividad antimicrobiana de quitosano y sus derivados se resumepor la siguiente secuencia de sucesos (1) adsorción del quitosano y/o oligómeros de éste sobre la superficie de la célula bacteriana, (2) difusión a través de la pared celular e interrupción de la función adecuada de ésta, (3) provocando la interrupción de la membrana citoplasmática, (5) y generando finalmente fugas de los constituyentes citoplásmicos, y (6) la muerte de la célula (Ikeda y Tazuke, 1984; Papineau et al., 1991).

\subsection{Otras aplicaciones del quitosano en alimentos de origen animal}

Carnes:

En productos cárnicos se ha utilizado el quitosano,por su alta actividad antimicrobiana,para controlar los principales patógenos involucrados en la transmisión de enfermedades a los seres humanos como se revisó anteriormente, sin embargo, las películas y soluciones de quitosano también se han utilizado debido a su eficacia sobre la estabilidad de almacenamiento de la carne y mejoramiento de sus propiedades organolépticas. Por ejemplo, Darmadji y Izumimoto (1994) observaron que la adición de $1 \% \mathrm{p} / \mathrm{v}$ de quitosano en carne de vacuno picada disminuyó significativamente el valor del indicador de ácido tiobarbitúrico (TBA) en comparación con la muestra control, demostrando que la adición de quitosano disminuye la oxidación de lípidos en la carne, 
resultando en un efecto deseable sobre la mantención del color rojo del productodurante el almacenamiento. Lee et al. (2003) observaron que trozos de carne de cerdo sumergida en soluciones de quitosano de peso molecular de 30y $120 \mathrm{kDa}$ al $1 \% \mathrm{p} / \mathrm{v}$, presentaron una mayor vida útil y menor oxidación lipídica.Park et al. (2010) también reportaron una mayor duración del color rojo característico de la carne vacuna al recubrirla con películas de polietileno de baja densidad y quitosano al $2 \% \mathrm{p} / \mathrm{v}$ en ácido láctico. Vargas et al. (2011) aplicaron películas comestibles basadas en quitosano en la superficie de hamburguesas de cerdo, logrando incrementar el contenido de metamioglobina, lo que se tradujo en un mejor aspecto de las hamburguesas.Otros autores también han reportado el uso del quitosano como antioxidante y han concluido que este efecto mejora la apariencia de la carne, relacionada con el color, y disminuye el olor desagradable generado por la rancidez de los lípidos propios de la carne (Kanattet al., 2004; Raoet al., 2005). Esto también ha sido descrito cuando películas de quitosano se han usado como recubrimiento comestible de filetes frescos de pescado (Jeon et al., 2002) y en camarones refrigerados (Huang et al., 2012).

Algunos investigadores reportaron en otros alimentos que el quitosano es capaz de establecer reacciones con la vitamina $\mathrm{E}$ mediante interacciones iónicas que la hacen más estable (Koide, 1998). Incluso según Pasanphan et al. (2010) recientemente el quitosano se ha comenzado a utilizar como una alternativa de antioxidante natural, debido a la capacidad de los oligómeros de soluciones de quitosano de atrapar radicales hidroxilos mediante reacciones iónicas con los grupos amino de su estructura química (Xie et al., 2001; Sun et al., 2007).

\section{Leche y productos lácteos}

En la literatura hay pocos estudios acerca de la utilización de quitosano para evaluar la posibilidaddel uso de quitosano para mejorar la calidad y vida útil de la leche. Leey Lee (2000) demostraron que la incorporación de soluciones de quitosano al 0,5\%y $1,0 \% \mathrm{p} / \mathrm{v}$ en leche líquida, podría mejorar el proceso de esterilización logrando hacerlo a $73^{\circ} \mathrm{C}$ durante $15 \mathrm{~s}$, evitando la coagulación de la proteína láctea. Sin embargo, la adición de quitosano afectó negativamente la calidad sensorial de la leche modificando el color, sabor y el aroma. Gammariello et al. (2008) estudiaron el efecto del quitosano sobre las propiedades sensoriales y reológicas de un queso untable durante su almacenamiento. En particular, el quitosano mejoró las propiedades antes mencionadas del queso para untar, siendo más suave que las muestras control y no afectó la microflora de éste. Fajardo et al. (2010) utilizaron películas de quitosano como base para impregnarnatamicina, y recubrieron con estas películas quesos semi-duros, los cuales presentaron un menor crecimiento fúngico (Aspergillus niger, PenicilliumcrustosumP. commune y $P$. roqueforti) en su superficie durante el almacenamiento. Di Pierroet al. (2011) aplicaron películas de mezcla entre quitosano/proteínas de suero de leche como envase activo a queso tipo ricota, y observaron una mayor vida útil de este producto y una disminución de los organismos mesófilos y psicotróficos.Del Nobileet al. (2009) utilizaron películas de quitosano solas y activas (impregnadas conlisozima, ácido etilendiaminatetraacético, sal disódica), combinadas con envasado en atmósferas modificadas, para prolongar la vida útil de quesos "Fior di Latte". Los autores encontraron que los quesos recubiertos almacenados obtuvieron una mayor vida considerable de anaquel que los quesos almacenados con envases tradicionales.

Por otra parte, existe un interés creciente en el desarrollo de nuevos productos con proteínas obtenidas desde los productos lácteos. Para este propósito, diferentes propiedades físicas y químicas de variados polímeros han sido utilizadas para aislar y recuperar proteínas de la leche (Huffman y Harper, 1999). Al respecto, el quitosano ha sido utilizado por la industria láctea, como agente coagulante de la caseína a través de interacciones electroestáticas e hidrofóbicas (Ausar et al., 2001). Como también debido a las propiedades mucoadhesivas del quitosano, que mejoran la adsorción de péptidos y estimulan la actividad de macrófagos y funciones inmunológicas (Nishimura et al., 1984; Rossi et al., 2000; Bianco et al., 2000). 
Otro uso potencial del quitosano es el desarrollo de nuevos productos lácteos fermentados, en los cuales se agrega este biopolímero como fibra natural, no obstante, se requiere que las bacterias representantes de la fermentación de la leche: L. delbrueckiissp. Bulgaricus, S. thermophilus y Propionibacteriumfreudenreichii puedan crecer en su presencia. En un estudio realizado por Ausaret al. (2002) lograron disminuir la actividad antimicrobiana del quitosano sobre éstas bacterias, debido a la fuerte interacción de los grupos catiónicos del quitosano con residuos de la caseína y/o grasa láctea, disminuyendo su acción antimicrobiana, y potenciando su efecto coagulante, logrando obtener un queso enriquecido con fibra.

Se ha reportado que el quitosano también disminuye las reacciones de deterioro de los alimentos cárnicos y lácteos, evitando el pardeamiento no enzimático (PNE) o reacción de Maillard, siendo este fenómeno nefasto para la calidad alimentaria, puesto que provoca la disminución del valor nutritivo y aparición de color y sabores desfavorables, y origina productos potencialmente tóxicos. El PNE se produce durante la preparación de alimentos líquidos concentrados como leche, la cocción de algunos alimentos marinos y la deshidratación de leche, huevos, carnes, harina de pescado, etc. La reducción del PNE de los alimentos antes mencionados se produciría por la reacción de los grupos amino del quitosano con los grupos carbonilo, de los azúcares reductores, algunas vitaminas, productos de la oxidación de lípidos y compuestos con grupos amino como los aminoácidos (Rodríguez et al., 2001; Rodríguez et al., 2002).

\section{Cecinas}

Los resultados indican que la aplicación de un recubrimiento con quitosano por inmersión de cecinas, mejora la calidad microbiológica y extiende la vida útil, lo que podría ser una alternativa a la protección química mediante aditivos(Bostan y Isin Mahan, 2011).

Huevos
Diversos problemas se han encontrado durante el almacenamiento de los huevos, tales como la pérdida de peso, deterioro de la calidad interior, y la contaminación microbiana (Bhale et al., 2003; De Reu et al., 2006). Algunos investigadores han reportado que el recubrir huevos con quitosano es eficaz en la preservación de la calidad interna de los huevos sin afectar a la aprobación del consumidor. Así por ejemplo, se ha probado la mejoría en la vida útil en estantería de huevos recubierto con quitosano (Caner, 2005). Por otra parte, las unidades Haugh y los valores de índice de yema muestran que la calidad de la albúmina y la yema de huevos revestidos con quitosano son preservadas hasta por 5 semanas a $25^{\circ} \mathrm{C}$, que es por lo menos 3 semanas más que el observado para huevos control (Bhale et al., 2003) además demostrar una significativa reducción de las pérdidas de pesode huevos tratados (Kim et al., 2006)

\section{Conclusión}

La amplia actividad antimicrobiana del quitosano junto con sus otras bondades (agente floculante, coagulante, antioxidante, disminuir ciertas reacciones que reducen la calidad de los alimentos), que afectan las características organolépticas de los alimentos, ha hecho que sea considerado como un buen conservante natural de productos alimenticios.El quitosano ha sido aprobado como un aditivo alimentario en Corea y Japón desde 1995 y 1983, respectivamente. En los Estados Unidos, ha recibido la aprobación de la FDA como aditivo alimentario y su aplicación en sistemas de alimentación será ciertamente un producto de demanda creciente en un futuro próximo en los productos de origen animal. Las necesidades y desafíos que se vislumbran para el futuro de la producción, procesamiento y exportación de productos de origen animal en Chile, junto con, la posibilidad de aprovechar de mejor manera las fuentes naturales de quitosano desde los desechos de la industria pesquera, podrían entregar a Chile una ventaja comparativa en la comercialización y penetración comercial de estos productos. Por otra parte, la investigación en aplicaciones innovadoras y más eficientes de usos del quitosano en la industria de los alimentos, generaría en 
Chile una nueva fuente de negocio de productos para la conservación de alimentos para la exportación.

\section{Referencia}

1. Aider, M. 2010. Chitosan application for active bio-based films production and potential in the food industry: Review. LWT-FoodSci. Technol. $43,837-842$.

2. Agulló, E.; Rodríguez, M.; Ramos, V.; Albertengo L. 2003. Present and future role of chitin and chitosan in food. Macromol. Biosci. 3, 521-230.

3. Albuquerque, R.; Montenegro, T.; Montenegro, T.; Cardoso, S.; Souza, E. 2011. Sensory evaluation and inhibition of Listeria monocytogenes in bovine pâté added of chitosan from Mucorrouxii. . LWTFood Sci. Technol. 44, 588-591.

4. Altieri, C.; Scrocco, C.; Sinigaglia, M.; Del Nobile, M. 2005. Use of chitosan to prolong mozzarella cheese shelf life. J. DairySci. 88, 26832688 .

5. Arias, J.; Neira-Carrillo, A.; Yazdani-Pedram, M.; Fernandez, M.; Arias, J. 2008. Phosphorylation efficiency of chitosan at different times and temperatures for bone graft substitute. TissueEng. 14(5), 895-896.

6. Ausar, S.; Bianco, I.; Badini, R.; Castagna, L.; Modesti, N.; Landa, C.; Beltramo, D.; 2001a. Characterization of casein micelle precipitation by chitosans. J. Dairy Sci. 84, 361-369.

7. Ausar, S.; Passalacqua, N.; Castagna, L.; Bianco, I.; Beltramo, D. 2002. Growth of milk fermentative bacteria in the presence of chitosan for potential use in cheese making. Int. Dairy J. 12, 899-906.

8. Beverlya, R.; Janes M.; Prinyawiwatkula, W.; No, H. 2008. Edible chitosan films on ready-to-eat roast beef for the control of Listeria monocytogenes. Food Microbiol.25, 534-537.

9. Begin, A.; Van Calsteren, M. 1999. Antimicrobial films produced from chitosan. Int. J. Biol. Macromol. 26 (1), 63-67.
10. Bhale, S.; No, H.; Prinyawiwatkul, W.; Farr, A.; Nadarajah, K.; Meyers, S. 2003. Chitosan coating improves shelf life of eggs. J. Food Sci. 68(7), 2378-2383.

11. Bianco, I.; Balsinde, J.; Beltramo, D.; Castagna, L.; Landa, C.; Dennis, E. 2000. Chitosan-induced phospholipase $\mathrm{A}_{2}$ activation and arachidonic acid mobilization in $\mathrm{P}^{388 \mathrm{D}_{\mathrm{I}}}$ macrophages. FEBS Letters. 466, 292-294.

12. Bostan, K.; Mahan, F. 2011. Microbiological quality and shelf-life of sausage treated with chitosan. J. Fac. Vet. Med. Istanbul Univ. 37(2), $117-126$

13. Butler, B.; Vergano, P.; Testin, R.; Bunn, J.; Wiles, J. 1996. Mechanical and barrier properties of edible chitosan films as affected by composition and storage. J. Food Sci. 61(5), 953-955.

14. Caner, C. 2005. The effect of edible eggshell coatings on egg quality and consumer perception. J. Sci. Food Agr. 85(11), 1897-1902.

15. Caner, C.; Vergano, P.; Wiles, J. 1998. Chitosan films: mechanical and permeation properties as affected by acid, plasticizer, and storage. J. Food Sci. 63(6), 1049-1053.

16. Chhabra, P.; Huang, Y.; Frank, J.; Chmielewski, R.; Gates, K. 2006. Fate of staphylococcus aureus, salmonella entericaserovartyphimurium, and vibrio vulnificus in raw oysters treated with chitosan. J. Food Protect. 69, 952-959.

17. Coma, V.; Gros, M.; Garreau, S.; Copinet, A.; Salin, F.; Deschamps, A. 2002. Edible antimicrobial films based on chitosan matrix. J. Food Sci. 67(3), 1162-1169.

18. Collins, J. 1997. Impact of changing consumer lifestyles on the emergence of and re-emergence of foodborne pathogens. Emerg. Infect. Dis. 3(4), 471-479.

19. Darmadji, P.; Izumimoto, M. 1994. Effects of chitosan and nitrite on the properties of fermented meat. Anim. Sci. Technol. 65(7), 639-646.

20. De Reu, K.; Grijspeerdt, K.; Messens, W.; Heyndrickx, M.; Uyttendaele, M.; Debevere, J.; Herman, L. 2006. Eggshell factors influencing eggshell penetration and whole egg contamination 
by different bacteria, including Salmonella enteritidis. Int. J. Food Microbiol. 112(3), 253260.

21. Del Nobile, M.; Gammariello, D.; Conte, A.; Attanasio, M. 2009. A combination of chitosan, coating and modified atmosphere packaging for prolonging "Fior di latte" cheese shelf life. Carbohyd. Polym. 78, 151-156.

22. Di Pierro, P.; Sorrentino, A.; Mariniello, L.; Giosafatto, C.; Porta, R. 2011. Chitosan/whey protein film as active coating to extend Ricotta cheese shelf-life. LWT-Food Sci. Technol. 44, 2324-2327.

23. Dinesh, S; Alok, R. 2000. Biomedical applications of chitin, chitosan, and their derivatives. Rev. Macromol. Chem. Phys. 40(1), 69-83.

24. Doyle, M. 1991. Escherichia coli O157:H7 and its significance in foods. Int. J. Food Microbiol. 12, 289-302.

25. Dutta, P.; Tripathi, S.; Mehrotra, G.; Dutta, J. 2009. Perspectives for chitosan based antimicrobial films in food applications. Food Chem. 114, 1173-1182.

26. EFSA. 2010. Analysis of the baseline survey on the prevalence of Campylobacter in broiler batches and of Campylobacter and Salmonella on broiler carcasses in the EU, 2008. Part A: Campylobacter and Salmonella prevalence estimates. EFSA J. 8, 1503.

27. European Commission. 2003. Regulation (EC) $n$ $1831 / 2003$ of the European Parliament and the Council of September 22, 2003 on additives for use in animal nutrition. Official Journal of the European Union, L268, 29-43.

28. FAO/WHO (Food and Agriculture Organization, World Health Organization), 2003. Hazard characterization for pathogens in food and water. Microbiological Risk Assessment Series, No. 3. Food and Agriculture Organization of the United Nations, VialedelleTerme di Caracalla, Rome, Italy.

29. Fajardo, P.; Martins, J.; Fuciños, C.; Pastrana, L.; Teixeira, J.; Vicente, A. 2010. Evaluation of a chitosan-based edible film as carrier of natamycin to improve the storability of Saloio cheese. J. FoodEng. 101, 349-356.

30. Fernandez-Saiz, P.; Soler, C.; Lagaron, J.; Ocio, M. 2010. Effects of chitosan films on the growth of Listeria monocytogenes, Staphylococcus aureus and Salmonella spp. in laboratory media and in fish soup. Int. J. Food Microbiol. 137, 287-294.

31. Franklin, T.; Snow, G. 1981. Biochemistry of antimicrobial action. London: Chapman \& Hall. p. 58.

32. Fujimoto, F.; Tsuchiya, Y.; Terao, M.; Nakamura, K.; Yamamoto, M. 2006. Antibacterial effects of Chitosan solution ${ }^{\circledR}$ against Legionella pneumophila, Escherichia coli, and Staphylococcus aureus. Int. J. FoodMicrobiol. 112, 96-101.

33. Ganan, M.; Carrascosa, A.; Martínez-Rodríguez, A. 2009. Antimicrobial activity of chitosan against Campylobacter spp. and other microorganisms and its mechanism of action. J. Food Prot. 72, 17351738.

34. Gammariello, D.; Chillo, S.; Mastromatteo, M.; Di Giulio, S.; Attanasio, M.; Del Nobile, M. 2008. Effect of chitosan on the rheological and sensorial characteristics of Apulia Spreadable Cheese. J. Dairy Sci. 91(11), 4155-4163.

35. Georgantelis, D.; Ambrosiadis, L.; Katikou, P.; Blekas, G.; Georgakis, S. 2007. Effect of rosemary extract, chitosan and $\alpha$-tocopherol on microbiological parameters and lipid oxidation of fresh pork sausages stored at $4^{\circ} \mathrm{C}$. Meat Sci. 76 , 172-181.

36. Gómez-Estaca, J.; López de Lacey, A.; LópezCaballero, M.; Gómez-Guillén, M.; Montero, P. 2010. Biodegradable gelatinechitosan films incorporated with essential oils as antimicrobial agents for fish preservation. Food Microbiol. 27, 889-896.

37. Ha, T.; Lee, S. 2001. Utilization of chitosan to improve the quality of processed milk. J. Korean. Soc. Food Sci. Nutr. 30(4), 630-4.

38. Helander, I.; Wright, A.; Mattila-Sandholm, T. 1997. Potential of lactic acid bacteria and novel antimicrobials against Gram-negative bacteria. Trends Food Sci.Tech. 8, 146-150. 
39. Helander, I.; Nurmiaho-Lassila, E.; Ahvenainen, R.; Rhoades, J.; Roller, S. 2001. Chitosan disrupts the barrier properties of the outer membrane of gram negative bacteria. Int. J. Food Microbiol. 71, 235-244.

40. Henriksen, I.; Green, K.; Smart, J.; Smistad, G; Karlsen, J. 1996. Bioadhesion of hydrated chitosans: an in vitro and in vivo study. Int. J. Pharm. 145, 231-40.

41. Hernandez-Lauzardo, A.; Bautista-Banos, S.; Velazquez-Del Valle, M.; Mendez-Montealvo, M.; Sanchez-Rivera, M.; Bello-Perez, L. 2008. Antifungal effects of chitosan with different molecular weights on in vitro development of Rhizopusstolonifer (Ehrenb.:Fr.) Vuill. Carbohyd. Polym. 73, 541-547.

42. Huang, J.; Chen, Q.; Qiu, M.; Li, S. 2012. Chitosan-based edible coatings for quality preservation of postharvest whiteleg shrimp (litopenaeusvannamei). J. Food Sci. 77(4), 491496.

43. Huffman, L.; Harper, W. 1999. Maximizing the value of milk through separation technologies. J. Dairy Sci. 82, 2238-2244.

44. Ikeda, T.; Tazuke, S. 1984. Biologically-active polycations.4. Synthesis and antimicrobial activity of poly(Trialkylvinylbenzylammonium chloride). Macromol. Chem. Phys. 185, 869-876.

45. Jeon, Y.; Kamil, J.; Shahidi, F. 2002. Chitosan as an edible invisible film for quality preservation of herring and Atlantic cod. J. Agr. Food Chem. 50(18), 5167-5178.

46. Jeon, Y.; Park, P.; Kim, S. 2001. Antimicrobial effect of chito-oligosaccharides produced by bioreactor. Carbohyd. Polym. 44, 71-76.

47. Juneja, V.; Thippareddi, H.; Bari, L.; Inatsu, Y.; Kawamoto, S.; Friedman, M. 2006. Chitosan protects cooked ground beef and turkey against Clostridium perfringens spores during chilling. J Food Sci. 71(6), 236-240.

48. Käferstein, F.; Motarjemi, Y.; Bettcher, D. 1997. Foodborne disease control: a transnational challenge. Emerg. Infect. Dis. 3(4), 503-510.
49. Kanatt, S.; Chander, R.; Sharma, A. 2008. Chitosan and mint mixture: A new preservative for meat and meat products. Food Chem. 107, 845852.

50. Kim, S.; No, H.; Kim, S.; Prinyawiwatkul, W. 2006. Effect of plasticizer concentration and solvent types on shelf-life of eggs coated with chitosan. J. Food Sci. 71(4), 349-353.

51. Koide, S. 1998. Chitin-chitosan: properties, benefits and risks. Nutr. Res. 18, 1091-1101.

52. Lahmer, R.; Williams, P.; Townsend, S.; Baker, S.; Jones, D. 2012. Antibacterial action of chitosan-arginine against Escherichia coli $\mathrm{O} 157$ in chicken juice. Food Cont. 26, 206-211.

53. Lee, H.; Park, S.; Ahn, D. 2003. Effect of storage properties of pork dipped in chitosan solution. J Korean Soc. Food Sci. Nutr. 32(4), 519-25.

54. Lee, J.; Lee, Y. 2000. The physico-chemical and sensory properties of milk with water soluble chitosan. Korean J. Food Sci. Technol. 32(4), 806813.

55. Lee, M.; Newell, D. 2006. Campylobacter in poultry: filling an ecological niche. Avian Dis. 50, $1-9$.

56. Leleu, S.; Herman, L.; Heyndrickx, M.; De Reu, K.; Michiels, C.; De Baerdemaeker, J.; Messens, W. 2011. Effects on Salmonella shell contamination and trans-shell penetration of coating hens' eggs with chitosan. Int. J. Food Microbiol. 145, 43-48.

57. Li, B.; Kennedy, J.; Peng, J.; Yie, X.; Xie, B. 2006. Preparation and performance evaluation of glucomannan-chitosan-nisin ternary antimicrobial blend film. Carbohyd. Polym. 65(4), 488-494.

58. Lin, K.-W., Chao, J.-Y., 2001. Quality characteristics of reduced-fat Chinese-style sausage as related to chitosan's molecular weight. Meat Science 59, 343-351.

59. Liu, N.; Chen, X.; Park, H.; Liu, C.; Liu, C.; Meng, X.; Yu, L. 2006. Effect of MW and concentration of chitosan on antibacterial activity of Escherichia coli. Carbohyd. Polym. 64, 60-65. 
60. López-Caballero, M.; Gómez-Guillén, M.; PérezMateos, M.; Montero, P. 2005. A chitosan-gelatin blend as a coating for fish patties. Food Hydrocolloid. 19, 303-311.

61. Majeti, N.; Kumar, R. 2000. A review: chitin and chitosan applications. React. Funct. Polym. 46(1), $1-27$.

62. Mead, P.; Slutsker, L.; Dietz, V.; McCaig, LF.;Bresee, J.; Shapiro, C.; Griffin, P.; Tauxe, R. 1999. Food-Related Illness and Death in the United States. Emerg. Infect. Dis. 5(5), 607-25.

63. Mellegård, H.; From, C.; Christensen, B.; Granum, P. 2011. Inhibition of Bacillus cereus spore outgrowth and multiplication by chitosan. Int. J. FoodMicrobiol. 149, 218-225.

64. Mengíbar, M.; Gañan, M.; Miralles, B.; Carrascosa, A.; Martínez-Rodríguez, A.; Peter, M.; Heras, A. 2011. Antibacterial activity of products of depolymerization of chitosans with lysozyme and chitosanase against Campylobacter jejuni. Carbohyd. Polym. 84, 844-848.

65. Nishimura, K.; Nishimura, S.; Nishi, N.; Saiki, I.; Tokura, S.; Azuma, I. 1984. Immunological activity of chitin and its derivatives. Vaccine. 2, 93-99.

66. No, H.; Meyers, S.; Prinyawiwatkul, W.; Xu, Z. 2007. Applications of Chitosan for Improvement of Quality and Shelf Life of Foods: A Review. J. Food Sci. 72(5), 87-100.

67. No, H.; Park, N.; Lee, S.; Meyers, S. 2002. Antibacterial activity of chitosans and chitosan oligomers with different molecular weights. Int. J. Food Microbiol. 74, 65-72.

68. Papineau, A.; Hoover, D.; Knorr, D.; Farkas, D. 1991. Antimicrobial effect of water-soluble chitosans with high hydrostatic pressure. Food Biotechnol. 5, 45-57.

69. Park, S.; Marsh, K.; Dawson, P. 2010. Application of chitosan-incorporated LDPE film to sliced fresh red meats for shelf life extension. Meat Sci. 85, 493-499.

70. Park, S.; Marsh, K.; Rhim, J. 2002. Characteristics of different molecular weight chitosan films, affected by the type of organic solvents. J. Food Sci. 67(1), 194-197.

71. Park, S.; Youn, S.; Kim, H.; Ahn, D. 1999. Studies on the improvement of storage property in meat sausage using chitosan-I. J. Korean Soc. Food Sci. Nutr. 28(1), 167-71.

72. Pasanphan, W.; Buettner, G.; Chirachanchai, S. 2010. Chitosan gallate as a novel potential polysaccharide antioxidant: an EPR study. Carbohyd. Res. 345, 132-140.

73. Petrou, S.; Tsiraki, M.; Giatrakou, V.; Savvaidis, I. 2012. Chitosan dipping or oregano oil treatments, singly or combined on modified atmosphere packaged chicken breast meat. Int. J. Food Microbiol. 156, 264-271.

74. Ouattara, B.; Simard, R.; Piette, G.; Bégin, A.; Holley, R. 2000. Inhibition of surface spoilage bacteria in processed meats by application of antimicrobial films prepared with chitosan. Int. J. Food Microbiol. 62, 139-48.

75. Raafat, D.; Sahl, H. 2009. Chitosan and its antimicrobial potential-a critical literature survey. Microb. Biotechnol. 2, 186-201.

76. Rabea, E.; Badawy, M.; Stevens, C.; Smagghe, G.; Steurbaut, W. 2003. Chitosan as antimicrobial agent: applications and mode of action. Biomacromol. 4(6), 1457-1465.

77. Rao, M.; Chander, R.; Sharma, A. 2005. Development of shelf-stable intermediate moisture meat products using active edible chitosan coating and irradiation. J. Food Sci. 70(7), 325-331.

78. Rhazi, M.; Desbrieres, J.; Tolaimate, A.; Alagui, A.; Vottero, P. 2004. Investigation of different natural sources of chitin: influence of the source and deacetylation process on the physicochemical characteristics of chitosan. PolymerInt. 49(4), 337344.

79. Rodríguez, M.; Centurión, M.; Ramos, V.; Agulló, E. 2001. Analytical determination of chitosan in food. In: T. Uragami, K. Kurita, T. Fukamizo (Eds.), Chitin and chitosan in life science. KodanshaScientific Ltd., Japan. p. 515.

80. Rodríguez, M.; Centurión, M.; Agulló, E. 2002. J. Food Sci. 67, 2576-2578. 
81. Romanazzi, G.; Nigro, F.; Ippolito, A.; Divenere, D.; Salerno, M. 2002. Effect of pre- and post harvest chitosan treatments to control storage grey mold of table grapes. J. Food Sci. 67(5), 18621867.

82. Rossi, S.; Ferrari, F.; Bonferoni, M.; Caramella, C. 2000. Characterization of chitosan hydrochloridemucin interaction by means of viscometric and turbidimetric measurements. Eur. J. Pharm. Sci. 10, 251-257.

83. Sagoo, S.; Board, R.; Roller, S. 2002. Chitosan inhibits growth of spoilage microorganisms in chilled pork products. Food Microbiol. 19(2-3), 175-182.

84. Sashiwa, H.; Saimoto, H.; Shigemasa, Y. 1990. Lysozyme susceptibility of partially deacetylated chitin. Int. J. Biol. Macromol. 12, 295-296.

85. Simpson, B.; Gagne, N.; Ashie, I.; Noroozi, E. 1997. Utilization of chitosan for preservation of raw shrimp (Pandalus borealis). Food Biotechnol. $11,25-44$.

86. Smith, H. 1997. Vero cytotoxin-producing Escherichia coli O157:H7 cause for concern. Soc. Gen. Microbiol. Q. 24, 54-55.

87. Shigemasa, Y.; Saito, K.; Sashiwa, H.; Saimoto, H. 1994. Enzymatic degradation of chitins and partially deacetylated chitins. Int. J. Biol. Macromol. 16(1), 43-9.

88. Subasingle, S. 1995. The development of crustacean and mollusc industries for chitin and chitosan resources. In: M. Zakaria, W. Wan-Muda, M. Abdullah (Eds.), Chitin and chitosan. PenerbitUniversitiKebangsaan., Malaysia. p. 2734.

89. Sun, T.; Zhou, D.; Xie, J.; Mao, F. 2007. Preparation of chitosan oligomers and their antioxidant activity. Eur. Food Res. Technol. 225, 451-456.

90. Tan, S.; Tan, T.; Wong, S.; Khor, E. 1996. The chitosan yield of zygomycetes at their optimum harvesting time. Carbohyd. Polym. 30(4), 239-242.
91. Tauxe, R. 1997. Emerging foodborne disease, an evolving public health challenge. Emerg. Infect. Dis. 3(4), 425-434.

92. Tharanathan, R.; Kittur, F.; 2003. Chitin-the undisputed biomolecule of great potential. Crit. Rev. Food Sci. 43(1), 61-87.

93. Tolaimate, A.; Desbriéres, J.; Rhazi, M.; Alagui, A.; Vincendon, M.; Vottero, P. 2000. On the influence of deacetylation process on the physicochemical characteristics of chitosan from squid chitin. Polymer. 41, 2463-2469.

94. Tsai, G.; Su, W.; Chen, H.; Pan, C. 2002. Antimicrobial activity of shrimp chitin and chitosan from different treatments and applications of fish preservation. Fish. Sci. 68, 170-177.

95. Tsai, G.; Su, W. 1999. Antibacterial activity of shrimp chitosan against Escherichia coli. J. Food Protect. 62, 239-243.

96. Vargas, M.; Albors, A.; Chiralt, A. 2011. Application of chitosan-sunflower oil edible films to pork meat hamburgers. Procedia Food Sci. 1, $39-43$.

97. Wang, G. 1992. Inhibition and inactivation of five species of foodborne pathogens by chitosan. Food Prot. 55, 916-919.

98. Wiles, J.; Vergano, P.; Barron, F.; Bunn, J.; Testin, R. 2000. Water vapor transmission rates and sorption behavior of chitosan films. J. Food Sci. 65(7), 1175-1179

99. Yang, T-C.; Li, C-F.; Chou, C-C. 2007. Cell age, suspending medium and metal ion influence the susceptibility of Escherichia coli O157:H7 to water-soluble maltose chitosan derivative. Int. J. Food Microbiol. 113, 258-262.

100.Ye, M.; Neetoo, H.; Chen, H. 2008a. Control of Listeria monocytogenes on ham steaks by antimicrobials incorporated into chitosan-coated plastic films. Food Microbiol. 25, 260-268.

101.Ye, M.; Neetoo, H.; Chen, H. 2008b. Effectiveness of chitosan-coated plastic films incorporating antimicrobials in inhibition of Listeria monocytogenes on cold-smoked salmon. Int. J. Food Microbiol. 127, 235-240. 
102.Youn, S.; Park, S.; Ahn, D. 2000. Studies on the improvement of storage property in meat sausage using chitosan. II Difference of storage property by molecular weight of chitosan. J. Korean Soc. Food Sci. Nutr. 29(5), 849-53.

103.Youn, S.; Park, S.; Kim, Y.; Ahn, D. 2001. Studies on substitution effect of chitosan against sodium nitrite in pork sausage. Korean J. Food Sci. Technol. 33(5), 551-559.

104.Xie, W.; Xu, P.; Liu, Q. 2001. Antioxidant activity of water-soluble chitosan derivates. Bioorg Med ChemLett. 11(13), 1699-1701.

105.Xing, K.; Chen, X.; Liu, C.; Cha, D.; Park, H. 2009. Oleoyl-chitosan nanoparticles inhibits Escherichia coli and Staphylococcus aureus by damaging the cell membrane and putative binding to extracellular or intracellular targets. Int. J. Food Microbiol. 132, 127-133.

106.Zakrzewska, A.; Boorsma, A.; Brul, S.; Hellingwerf, K.; Klis, F. 2005. Transcriptional response of Saccharomyces cerevisiae to the plasma membrane-perturbing compound chitosan. Eukaryot. Cell. 4, 703-715

107.Zakrzewska, A.; Boorsma, A.; Delneri, D.; Brul, S.; Oliver, S.; Klis, F. 2007. Cellular processes and pathways that protect Saccharomyces cerevisiae cells against the plasma membrane-perturbing compound chitosan. Eukaryot. Cell 6, 600-608.

108.Zivanovic, S.; Shuang, C.; Draughon, F. 2004. Antimicrobial activity of chitosan films enriched with essentials oils. J. Food Sci. 70, 45-51. 